\title{
Polysubstituted Isoflavonoids from Spatholobus suberectus, Flemingia macrophylla, and Cudrania cochinchinensis
}

\author{
Li-Xia Wang $\cdot$ Hai-Rong Zheng $\cdot$ Fu-Cai Ren $\cdot$ \\ Tian-Ge Chen $\cdot$ Xiang-Mei Li $\cdot$ Xian-Jun Jiang $\cdot$ \\ Fei Wang
}

Received: 15 December 2016/Accepted: 10 January 2017/Published online: 21 January 2017

(C) The Author(s) 2017. This article is published with open access at Springerlink.com

\begin{abstract}
Four hitherto unknown polysubstituted isoflavonoids, including three isoflavans: 7,4'-dihydroxy-8, $2^{\prime}, 3^{\prime}$ trimethoxyisoflavan (1), 7,2',4'-trihydroxy-8,3'-dimethoxyisoflavan (2), and 7,2',4'-trihydroxy-5-methoxyisoflavan (3), and one prenylated isoflavone cudraisoflavone M (4) were isolated from the ethanol extracts of Spatholobus suberectus (for 1 and 2), Flemingia macrophylla (for 3), and Cudrania cochinchinensis (for 4), respectively. Their structures were established on the basis of extensive spectroscopic analysis. Compounds $\mathbf{1}$ and $\mathbf{4}$ exhibited weak cytotoxic activity against five human cancer cell lines (HL-60, A-549, SMMC-7721, MCF-7, and SW-480).
\end{abstract}

Graphical Abstract
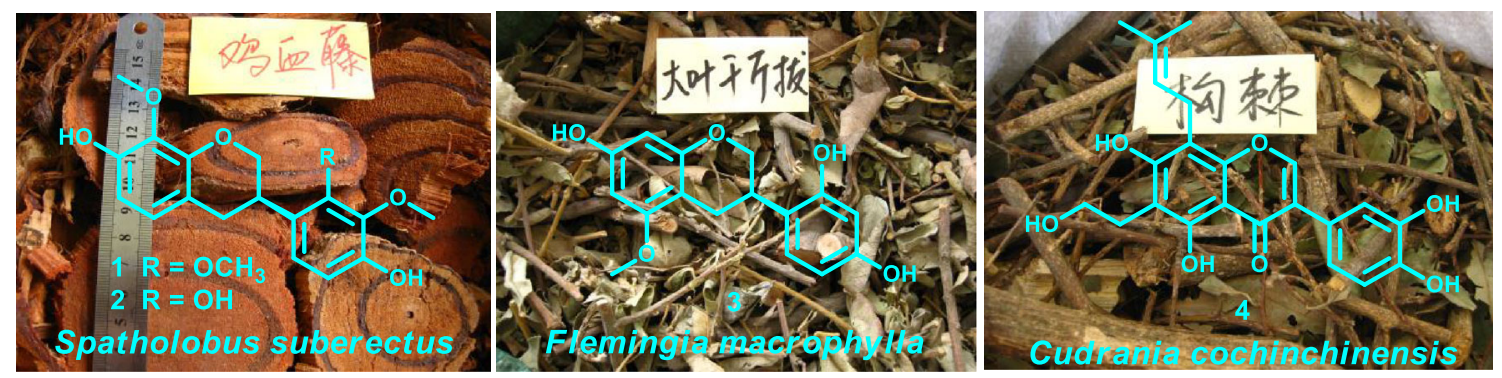

Keywords Spatholobus suberectus - Flemingia macrophylla .

Cudrania cochinchinensis · Isoflavan · Isoflavone · Cytotoxicity

\section{Introduction}

Isoflavonoids are a large group of secondary metabolites with diverse biological activities that occur widely in

Electronic supplementary material The online version of this article (doi:10.1007/s13659-017-0121-2) contains supplementary material, which is available to authorized users.

L.-X. Wang · H.-R. Zheng · F.-C. Ren · T.-G. Chen ·

X.-M. Li · X.-J. Jiang · F. Wang $(\bowtie)$

BioBioPha Co., Ltd., Kunming 650201,

People's Republic of China

e-mail: f.wang@mail.biobiopha.com plants, which can be subdivided into isoflavones, isoflavans, isoflavanones, rotenoids, pterocarpans, etc. An overwhelming number of isoflavonoids reported come from the Leguminosae family, and some non-leguminous families such as Moraceae are also relatively abundant in isoflavonoids [1, 2]. Some isoflavans and prenylated isoflavones possess potent biological activities, especially in cytotoxicity [3-6], anti-inflammatory [7], and neuroprotective activity [8]. As part of a BioBioPha (http://www. chemlib.cn) objective to assemble a large-scale natural product library valuable in the discovery of new drug leads from nature [9-12], the phytochemical investigations on Spatholobus suberectus (Leguminosae), Flemingia 
macrophylla (Leguminosae), and Cudrania cochinchinensis (Moraceae) led to the isolation of three new isoflavans $7,4^{\prime}$-dihydroxy-8,2',3'-trimethoxyisoflavan (1), 7,2',4'-trihydroxy-8,3'-dimethoxyisoflavan (2), and 7,2',4'-trihydroxy-5-methoxyisoflavan (3), and one new prenylated isoflavone namely cudraisoflavone $M$ (4), respectively (Fig. 1). Herein we report the structure elucidation of new isoflavonoid and their cytotoxicity evaluation against five human cancer cell lines (HL-60, A-549, SMMC-7721, MCF-7, and SW-480).

\section{Results and Discussion}

Compound 1 was obtained as white amorphous powder, and its molecular formula was determined to be $\mathrm{C}_{18} \mathrm{H}_{20} \mathrm{O}_{6}$ from the positive HRESIMS at $m / z 355.1163[\mathrm{M}+\mathrm{Na}]^{+}$ (calcd. for $\mathrm{C}_{18} \mathrm{H}_{20} \mathrm{O}_{6} \mathrm{Na}, 355.1158$ ) with nine degrees of unsaturation. The ${ }^{1} \mathrm{H}$ NMR spectrum (Table 1) displayed five aliphatic proton signals due to two methylene groups $\left[\delta_{\mathrm{H}} 2.86(1 \mathrm{H}, \mathrm{ddd}, J=15.8,5.3,1.9 \mathrm{~Hz})\right.$ and $2.94(1 \mathrm{H}, \mathrm{dd}$, $J=15.8,11.3 \mathrm{~Hz}), 4.00(1 \mathrm{H}, \mathrm{t}, J=10.5 \mathrm{~Hz})$ and 4.37 $(1 \mathrm{H}, \mathrm{ddd}, J=10.5,3.7,1.9 \mathrm{~Hz})]$, and one methine $\left[\delta_{\mathrm{H}}\right.$ $3.53(1 \mathrm{H}$, dddd, $J=11.3,10.5,5.3,3.7 \mathrm{~Hz})]$, which were characteristic of an isoflavan [13]. The ${ }^{1} \mathrm{H}$ NMR spectrum also exhibited two pairs of ortho-coupled aromatic doublets $\left[\delta_{\mathrm{H}} 6.52(1 \mathrm{H}, \mathrm{d}, J=8.3 \mathrm{~Hz}), 6.72(1 \mathrm{H}, \mathrm{d}\right.$, $J=8.3 \mathrm{~Hz}), 6.71(1 \mathrm{H}, \mathrm{d}, J=8.5 \mathrm{~Hz})$, and $6.75(1 \mathrm{H}, \mathrm{d}$, $J=8.5 \mathrm{~Hz})]$, three methoxy groups $\left[\delta_{\mathrm{H}} 3.87,3.92\right.$ and 3.93 (each $3 \mathrm{H}, \mathrm{s})]$, and two phenolic hydroxy protons $\left[\delta_{\mathrm{H}}\right.$ 5.66 and 5.71 (each $1 \mathrm{H}, \mathrm{s})]$. The ${ }^{13} \mathrm{C}$ NMR spectrum (Table 2) showed a total of 18 carbon signals, including 12 aromatic carbons for two phenyl units (rings $\mathrm{A}$ and $\mathrm{B}$ ), the ring $\mathrm{C}$ carbons at $\delta_{\mathrm{C}} 31.4(\mathrm{~d}), 31.5(\mathrm{t}), 70.5(\mathrm{t})$, and three methoxy carbons at $\delta_{\mathrm{C}} 60.6,60.8,61.0$ (each q). The above NMR spectroscopic features were very similar to those of isoduartin (=7,2'-dihydroxy-8, $3^{\prime}, 4^{\prime}$-trimethoxyisoflavan $)$ [14], except for an obvious downfield shift for $\mathrm{H}-5^{\prime}$ $(\Delta=+0.27 \mathrm{ppm})$, which hinted a different substituted pattern in ring $\mathrm{B}$. The pattern can be determined as $4^{\prime}$ hydroxy-2', $3^{\prime}$-dimethoxy by the following $\mathrm{HMBC}$ correlations (Fig. 2): from $4^{\prime}-\mathrm{OH}\left(\delta_{\mathrm{H}} 5.71\right)$ to $\mathrm{C}-3^{\prime}\left(\delta_{\mathrm{C}} 139.8\right)$, $\mathrm{C}-4^{\prime}\left(\delta_{\mathrm{C}} 148.5\right)$ and $\mathrm{C}-5^{\prime}\left(\delta_{\mathrm{C}} 110.5\right)$; from $\mathrm{H}-3\left(\delta_{\mathrm{H}} 3.53\right)$ and $2^{\prime}-\mathrm{OCH}_{3}\left(\delta_{\mathrm{H}} 3.87\right)$ to $\mathrm{C}-2^{\prime}\left(\delta_{\mathrm{C}} 150.8\right)$; and from $3^{\prime}-$ $\mathrm{OCH}_{3}\left(\delta_{\mathrm{H}} 3.93\right)$ to $\mathrm{C}-3^{\prime}$. The particular downfield shifts $(\Delta \approx+5 \mathrm{ppm})$ of aromatic methoxy carbons also implied the location of these groups. According to the empirical rule, the carbon signals of the methoxy groups with substituents in both ortho positions will appear at 60-62 ppm, while those sterically non-hindered at 55-57 ppm [15]. On the basis of the above analysis, the structure of $\mathbf{1}$ was established as 7,4'-dihydroxy-8, $2^{\prime}, 3^{\prime}$-trimethoxyisoflavan.

Compound 2, isolated as white amorphous powder, had a molecular formula of $\mathrm{C}_{17} \mathrm{H}_{18} \mathrm{O}_{6}$ by the positive HRESIMS at $m / z \quad 341.1002 \quad[\mathrm{M}+\mathrm{Na}]^{+}$(calcd. for $\mathrm{C}_{17} \mathrm{H}_{18} \mathrm{O}_{6} \mathrm{Na}$, 341.1001). Comparison of the ${ }^{1} \mathrm{H}$ and ${ }^{13} \mathrm{C}$ NMR spectral data (Tables 1,2) with those of $\mathbf{1}$ revealed that a methoxy group in the ring $\mathrm{B}$ was replaced by a hydroxy. From the HMBC correlations of the $\mathrm{OH}$ signal at $\delta_{\mathrm{H}} 5.72(1 \mathrm{H}, \mathrm{s})$ to $\mathrm{C}-1^{\prime}\left(\delta_{\mathrm{C}} 119.9\right), \mathrm{C}-2^{\prime}\left(\delta_{\mathrm{C}} 147.1\right)$, and $\mathrm{C}-3^{\prime}\left(\delta_{\mathrm{C}} 134.5\right)$, we could conclude that the emerging hydroxy was positioned at C-2' (Fig. 2). Hence, the structure of 2 was established as $7,2^{\prime}, 4^{\prime}$-trihydroxy-8,3'-dimethoxyisoflavan.

Compound 3 was isolated as white amorphous powder, and its molecular formula was determined to be $\mathrm{C}_{16} \mathrm{H}_{16} \mathrm{O}_{5}$ according to the positive HRESIMS at $\mathrm{m} / \mathrm{z} 289.1075$ $[\mathrm{M}+\mathrm{H}]^{+}$(calcd. for $\mathrm{C}_{16} \mathrm{H}_{17} \mathrm{O}_{5}, 289.1076$ ). Five diagnostic ring $\mathrm{C}$ proton signals $\left[\delta_{\mathrm{H}} 2.59(1 \mathrm{H}, \mathrm{dd}, J=16.3,10.8 \mathrm{~Hz})\right.$, $2.76(1 \mathrm{H}, \mathrm{ddd}, J=16.3,5.5,1.9 \mathrm{~Hz}), 3.34$ (1H, dddd, $J=10.8,10.2,5.5,3.1 \mathrm{~Hz}), 3.86(1 \mathrm{H}, \mathrm{dd}, J=10.2$, $10.2 \mathrm{~Hz})$, and $4.16(1 \mathrm{H}$, ddd, $J=10.2,3.1,1.9 \mathrm{~Hz})]$, an ABX-type aromatic proton system $\left[\delta_{\mathrm{H}} 6.30(1 \mathrm{H}, \mathrm{d}\right.$, $J=2.4 \mathrm{~Hz}), 6.24(1 \mathrm{H}, \mathrm{dd}, J=8.3,2.4 \mathrm{~Hz})$, and $6.85(1 \mathrm{H}$, $\mathrm{d}, J=8.3 \mathrm{~Hz})]$, two meta-coupled aromatic doublets $\left[\delta_{\mathrm{H}}\right.$ $5.88(1 \mathrm{H}, \mathrm{d}, J=2.1 \mathrm{~Hz})$ and $5.99(1 \mathrm{H}, \mathrm{d}, J=2.1 \mathrm{~Hz})]$, and one methoxy signal $\left[\delta_{\mathrm{H}} 3.74(3 \mathrm{H}, \mathrm{s})\right]$ were observed in the ${ }^{1} \mathrm{H}$ NMR spectrum (Table 1), which revealed that 3 was

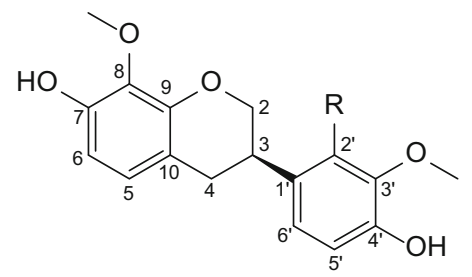

$1 \mathrm{R}=\mathrm{OCH}_{3}$

$2 \mathrm{R}=\mathrm{OH}$

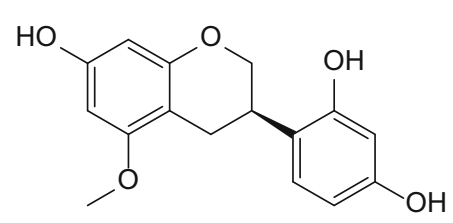

3<smiles>[B-]C([CH2-])=C[CH+]c1c(O)c([12CH2]CO)c(O)c2c(=O)c(-c3ccc(O)c(O)c3)coc12</smiles>

4

Fig. 1 Structures of new compounds 1-4 
Table $1{ }^{1}$ H NMR spectroscopic data of compounds 1-3

\begin{tabular}{llll}
\hline No. & $\mathbf{1}^{\mathrm{a}}$ & $\mathbf{2}^{\mathrm{a}}$ & $\mathbf{3}^{\mathrm{b}}$ \\
\hline $2-\mathrm{H}_{e q}$ & $4.37(\mathrm{ddd}, 10.5,3.7,1.9)$ & $4.43(\mathrm{ddd}, 10.2,3.4,2.0)$ & $4.16(\mathrm{ddd}, 10.2,3.1,1.9)$ \\
$2-\mathrm{H}_{a x}$ & $4.00(\mathrm{dd}, 10.5,10.5)$ & $4.07(\mathrm{dd}, 10.2,10.2)$ & $3.86(\mathrm{dd}, 10.2,10.2)$ \\
3 & $3.53(\mathrm{dddd}, 11.3,10.5,5.3,3.7)$ & $3.52(\mathrm{dddd}, 10.9,10.2,5.3,3.4)$ & $2.59(\mathrm{dd}, 16.3,10.8)$ \\
$4-\mathrm{H}_{a x}$ & $2.94(\mathrm{dd}, 15.8,11.3)$ & $3.01(\mathrm{dd}, 15.8,10.9)$ & $2.76(\mathrm{ddd}, 16.3,5.5,1.9)$ \\
$4-\mathrm{H}_{e q}$ & $2.86(\mathrm{ddd}, 15.8,5.3,1.9)$ & $2.90(\mathrm{ddd}, 15.8,5.3,2.0)$ & $5.99(\mathrm{~d}, 2.1)$ \\
5 & $6.72(\mathrm{~d}, 8.3)$ & $6.72(\mathrm{~d}, 8.3)$ & $5.88(\mathrm{~d}, 2.1)$ \\
6 & $6.52(\mathrm{~d}, 8.3)$ & $6.52(\mathrm{~d}, 8.3)$ & $6.30(\mathrm{~d}, 2.4)$ \\
8 & & & $6.24(\mathrm{dd}, 8.3,2.4)$ \\
$3^{\prime}$ & & $6.85(\mathrm{~d}, 8.3)$ \\
$5^{\prime}$ & $6.71(\mathrm{~d}, 8.5)$ & $6.74(\mathrm{~d}, 8.5)$ & \\
$6^{\prime}$ & $6.75(\mathrm{~d}, 8.5)$ & $5.69(\mathrm{~s})$ & $3.74(\mathrm{~s})$ \\
$7-\mathrm{OH}^{\prime}-\mathrm{OH}^{\prime}$ & $5.66(\mathrm{~s})$ & $5.72(\mathrm{~s})$ & \\
$4^{\prime}-\mathrm{OH}$ & & $5.30(\mathrm{br} \mathrm{s})$ & \\
$5-\mathrm{OCH}$ & & $3.91(\mathrm{~s})$ & \\
$8-\mathrm{OCH}_{3}$ & $5.71(\mathrm{~s})$ & $3.88(\mathrm{~s})$ & \\
$2^{\prime}-\mathrm{OCH}_{3}$ & $3.92(\mathrm{~s})$ & & \\
$3^{\prime}-\mathrm{OCH}_{3}$ & $3.87(\mathrm{~s})$ & & \\
\hline
\end{tabular}

${ }^{a}$ Measured in $\mathrm{CDCl}_{3}\left(\delta_{\mathrm{H}} 7.26 \mathrm{ppm}\right)$

b Measured in $\mathrm{CD}_{3} \mathrm{OD}\left(\delta_{\mathrm{H}} 3.30 \mathrm{ppm}\right)$

Table $2{ }^{13} \mathrm{C}$ NMR spectroscopic data of compounds $\mathbf{1}-\mathbf{3}$

\begin{tabular}{lllr}
\hline No. & $\mathbf{1}^{\mathrm{a}}$ & $\mathbf{2}^{\mathrm{a}}$ & \multicolumn{1}{c}{$\mathbf{3}^{\mathrm{b}}$} \\
\hline 2 & $70.5(\mathrm{t})$ & $69.8(\mathrm{t})$ & $71.0(\mathrm{t})$ \\
3 & $31.4(\mathrm{~d})$ & $31.8(\mathrm{~d})$ & $32.6(\mathrm{~d})$ \\
4 & $31.5(\mathrm{t})$ & $30.3(\mathrm{t})$ & $26.1(\mathrm{t})$ \\
5 & $124.3(\mathrm{~d})$ & $124.3(\mathrm{~d})$ & $160.1(\mathrm{~s})$ \\
6 & $107.0(\mathrm{~d})$ & $106.9(\mathrm{~d})$ & $92.3(\mathrm{~d})$ \\
7 & $147.5(\mathrm{~s})$ & $147.4(\mathrm{~s})$ & $157.7(\mathrm{~s})$ \\
8 & $134.8(\mathrm{~s})$ & $134.7(\mathrm{~s})$ & $96.5(\mathrm{~d})$ \\
9 & $147.1(\mathrm{~s})$ & $147.1(\mathrm{~s})$ & $156.9(\mathrm{~s})$ \\
10 & $115.3(\mathrm{~s})$ & $115.3(\mathrm{~s})$ & $104.1(\mathrm{~s})$ \\
$1^{\prime}$ & $126.3(\mathrm{~s})$ & $119.9(\mathrm{~s})$ & $120.4(\mathrm{~s})$ \\
$2^{\prime}$ & $150.8(\mathrm{~s})$ & $147.1(\mathrm{~s})$ & $157.2(\mathrm{~s})$ \\
$3^{\prime}$ & $139.8(\mathrm{~s})$ & $134.5(\mathrm{~s})$ & $103.5(\mathrm{~d})$ \\
$4^{\prime}$ & $148.5(\mathrm{~s})$ & $147.4(\mathrm{~s})$ & $157.9(\mathrm{~s})$ \\
$5^{\prime}$ & $110.5(\mathrm{~d})$ & $107.8(\mathrm{~d})$ & $107.6(\mathrm{~d})$ \\
$6^{\prime}$ & $122.0(\mathrm{~d})$ & $122.7(\mathrm{~d})$ & $128.8(\mathrm{~d})$ \\
$5-\mathrm{OCH}_{3}$ & & & $55.8(\mathrm{q})$ \\
$8-\mathrm{OCH}_{3}$ & $61.0(\mathrm{q})$ & $60.9(\mathrm{q})$ & \\
$2^{\prime}-\mathrm{OCH}_{3}$ & $60.8(\mathrm{q})$ & & \\
$3^{\prime}-\mathrm{OCH}_{3}$ & $60.6(\mathrm{q})$ & $61.2(\mathrm{q})$ & \\
\hline & & &
\end{tabular}

${ }^{\text {a }}$ Measured in $\mathrm{CDCl}_{3}\left(\delta_{\mathrm{C}} 77.0 \mathrm{ppm}\right)$

b Measured in $\mathrm{CD}_{3} \mathrm{OD}\left(\delta_{\mathrm{C}} 49.0 \mathrm{ppm}\right)$

a monomethyl ether derivative of $5,7,2^{\prime}, 4^{\prime}$-tetrahydroxyisoflavan. The HMBC correlations from the methoxy and $\mathrm{H}-4$ proton signals to $\mathrm{C}-5\left(\delta_{\mathrm{C}} 160.1\right)$ confirmed the
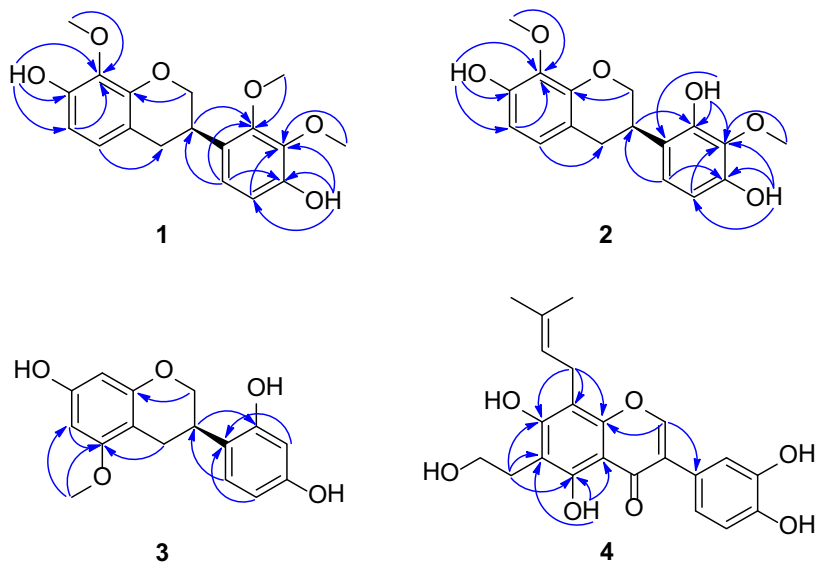

Fig. 2 Key HMBC ( $)$ correlations of 1-4

methoxy group at C-5 (Fig. 2). Therefore, the structure of 3 was established as 7,2',4'-trihydroxy-5-methoxyisoflavan.

Absolute configurations of the isoflavans (1-3) were established by comparison of the CD curves with those reported earlier for closely related analogues. All the CD spectra showed a negative Cotton effect in the transition region $(215-240 \mathrm{~nm})$, similar with those of 7-O-methylisomucronulatol, (3R)-isomucronulatol, and abruquinone L [16-19], which indicated the absolute configurations at C-3 of 1-3 being $R$-form. And optical rotation measurements were also taken to assign the absolute configuration. We carefully examined the specific rotation 
values of some $2^{\prime}, 3^{\prime}, 4^{\prime}$-trisubstituted isoflavans (Table 3 ), and the results suggested that the methoxy group substituted at $\mathrm{C}-2^{\prime}$ had a huge impact on the specific rotation, probably on account of the spatial proximity. For $2^{\prime}$-methoxyisoflavans, $R$-/ $S$-form result in positive/negative specific rotation values, respectively, but for $2^{\prime}$-hydroxyisoflavans, $R$-form gives negative values [14, 17, 20-25]. Our current research results were consistent with the above empirical rule.

Compound 4, yellowish amorphous powder, had a molecular formula of $\mathrm{C}_{22} \mathrm{H}_{22} \mathrm{O}_{7}$ according to the positive HRESIMS at $m / z$ 399.1440 $[\mathrm{M}+\mathrm{H}]^{+}$(calcd. for $\mathrm{C}_{22} \mathrm{H}_{23} \mathrm{O}_{7}$, 399.1444). The NMR spectra (Table 4) showed an olefinic signal $\left(\delta_{\mathrm{H}} 8.23,1 \mathrm{H}, \mathrm{s} ; \delta_{\mathrm{C}} 154.3, \mathrm{~d}\right)$ and a chelated hydroxy group at $\delta_{\mathrm{H}} 13.48(1 \mathrm{H}, \mathrm{s})$, characteristic of a 5-hydroxyisoflavone skeleton [8, 13]. In addition, an ABX-type aromatic proton system $\left[\delta_{\mathrm{H}} 7.15(1 \mathrm{H}, \mathrm{d}, J=2.0 \mathrm{~Hz}), 6.86\right.$ $(1 \mathrm{H}, \mathrm{d}, J=8.2 \mathrm{~Hz})$, and $6.94(1 \mathrm{H}, \mathrm{dd}, J=8.2,2.0 \mathrm{~Hz})]$, a prenyl group $\left[\delta_{\mathrm{H}} 1.63,1.79\right.$ (each $\left.3 \mathrm{H}, \mathrm{s}\right), 3.45(2 \mathrm{H}, \mathrm{d}$, $J=7.1 \mathrm{~Hz})$, and $5.22(1 \mathrm{H}$, br t, $J=7.1 \mathrm{~Hz})]$, and a hydroxyethyl moiety $\left[\delta_{\mathrm{H}} 2.97,3.90\right.$ (each $2 \mathrm{H}, \mathrm{t}$, $J=5.2 \mathrm{~Hz}$ )] were also detected in the ${ }^{1} \mathrm{H}$ NMR spectrum. The ${ }^{13} \mathrm{C}$ NMR spectrum displayed a total of 22 carbon resonances, including two methyls, three $s p^{3}$ methylenes, five $s p^{2}$ methines, and $12 s p^{2}$ quaternary carbons. These spectroscopic features were very similar to those of cudraisoflavone $\mathrm{L}$, which was recently isolated from the same genus and shared the same molecular formula with 4 [26]. Their structural difference was only due to the location of the prenyl group and the hydroxyethyl moiety. The hydroxyethyl unit was located at C-6 from the HMBC correlations of $\mathrm{H}-1^{\prime \prime}\left(\delta_{\mathrm{H}} 2.97\right)$ and 5-OH $\left(\delta_{\mathrm{H}} 13.48\right)$ to C-5 $\left(\delta_{\mathrm{C}} 158.5\right)$ and $\mathrm{C}-6\left(\delta_{\mathrm{C}} 111.2\right)$, while the prenyl group at C-8 by the correlations of $\mathrm{H}-1^{\prime \prime \prime}\left(\delta_{\mathrm{H}} 3.45\right)$ and $\mathrm{H}-2\left(\delta_{\mathrm{H}}\right.$ $8.23)$ to $\mathrm{C}-9\left(\delta_{\mathrm{C}}\right.$ 154.6). Thus the structure of 4 was established and named as cudraisoflavone $\mathrm{M}$. It is worth mentioning that cudraisoflavone M (4) was also obtained in our phytochemical investigation on the plant Derris robusta (Leguminosae).

Table 3 Specific rotation values of some $2^{\prime}, 3^{\prime}, 4^{\prime}$-trisubstituted isoflavans

\begin{tabular}{|c|c|c|c|c|c|c|c|c|c|}
\hline \multirow[t]{2}{*}{ Compound } & \multicolumn{7}{|c|}{ Substitution pattern } & \multirow[t]{2}{*}[\alpha]{$_{D}$} & \multirow[t]{2}{*}{ References } \\
\hline & 3 & 6 & 7 & 8 & $2^{\prime}$ & $3^{\prime}$ & $4^{\prime}$ & & \\
\hline (-)-Duartin & $S$ & - & $\mathrm{OH}$ & $\mathrm{OMe}$ & $\mathrm{OMe}$ & $\mathrm{OH}$ & $\mathrm{OMe}$ & $-18.5\left(\mathrm{CHCl}_{3}\right)$ & [20] \\
\hline Mucronulatol & $S$ & - & $\mathrm{OH}$ & - & $\mathrm{OMe}$ & $\mathrm{OH}$ & $\mathrm{OMe}$ & $-18.5\left(\mathrm{Me}_{2} \mathrm{CO}\right)$ & [21] \\
\hline Bryaflavan & $S$ & $\mathrm{OH}$ & $\mathrm{OH}$ & - & $\mathrm{OMe}$ & $\mathrm{OH}$ & $\mathrm{OMe}$ & $-17.3(\mathrm{MeOH})$ & [22] \\
\hline 2'-O-Methylisomucronulatol & $R$ & - & $\mathrm{OH}$ & - & $\mathrm{OMe}$ & $\mathrm{OMe}$ & $\mathrm{OMe}$ & $+6.5\left(\mathrm{CHCl}_{3}\right)$ & [23] \\
\hline$(+)$-Duartin & $R$ & - & $\mathrm{OH}$ & $\mathrm{OMe}$ & $\mathrm{OMe}$ & $\mathrm{OH}$ & $\mathrm{OMe}$ & $+3.4\left(\mathrm{CHCl}_{3}\right)$ & [14] \\
\hline 8,3'-Dihydroxyvestitol & $R$ & - & $\mathrm{OH}$ & $\mathrm{OH}$ & $\mathrm{OH}$ & $\mathrm{OH}$ & $\mathrm{OMe}$ & $-5.2(\mathrm{MeOH})$ & [24] \\
\hline Isomucronulatol & $R$ & - & $\mathrm{OH}$ & - & $\mathrm{OH}$ & $\mathrm{OMe}$ & $\mathrm{OMe}$ & $-19.4(\mathrm{EtOH})$ & [25] \\
\hline 7-O-Methylisomucronulatol & $R$ & - & $\mathrm{OMe}$ & - & $\mathrm{OH}$ & $\mathrm{OMe}$ & $\mathrm{OMe}$ & $-11.0\left(\mathrm{CHCl}_{3}\right)$ & [17] \\
\hline
\end{tabular}

Table 4 NMR spectroscopic data of cudraisoflavone M (4) in acetone- $d_{6}\left(\delta_{\mathrm{H}} 2.04 \mathrm{ppm}, \delta_{\mathrm{C}} 29.8 \mathrm{ppm}\right)$

\begin{tabular}{|c|c|c|c|c|c|}
\hline No. & $\delta_{\mathrm{H}}$ & $\delta_{\mathrm{C}}$ & No. & $\delta_{\mathrm{H}}$ & $\delta_{\mathrm{C}}$ \\
\hline 2 & $8.23(\mathrm{~s})$ & $154.3(\mathrm{~d})$ & $4^{\prime}$ & & $146.1(\mathrm{~s})$ \\
\hline 3 & & $123.5(\mathrm{~s})$ & $5^{\prime}$ & $6.86(\mathrm{~d}, 8.2)$ & $115.8(d)$ \\
\hline 4 & & $182.0(\mathrm{~s})$ & $6^{\prime}$ & $6.94(\mathrm{dd}, 8.2,2.0)$ & $121.4(\mathrm{~d})$ \\
\hline 5 & & $158.5(\mathrm{~s})$ & $1^{\prime \prime}$ & $2.97(\mathrm{t}, 5.2)$ & $26.1(\mathrm{t})$ \\
\hline 6 & & $111.2(\mathrm{~s})$ & $2^{\prime \prime}$ & $3.90(t, 5.2)$ & $63.7(t)$ \\
\hline 7 & & $161.8(\mathrm{~s})$ & $1^{\prime \prime \prime}$ & $3.45(\mathrm{~d}, 7.1)$ & $22.4(\mathrm{t})$ \\
\hline 8 & & $107.7(\mathrm{~s})$ & $2^{\prime \prime \prime}$ & $5.22($ br t, 7.1$)$ & $123.3(d)$ \\
\hline 9 & & $154.6(\mathrm{~s})$ & $3^{\prime \prime \prime}$ & & $131.8(\mathrm{~s})$ \\
\hline 10 & & $105.8(\mathrm{~s})$ & $4^{\prime \prime \prime}$ & $1.63(\mathrm{~s})$ & $25.8(\mathrm{q})$ \\
\hline $1^{\prime}$ & & $123.8(\mathrm{~s})$ & $5^{\prime \prime \prime}$ & $1.79(\mathrm{~s})$ & $17.9(\mathrm{q})$ \\
\hline $2^{\prime}$ & $7.15(\mathrm{~d}, 2.0)$ & $117.2(\mathrm{~d})$ & $5-\mathrm{OH}$ & $13.48(\mathrm{~s})$ & \\
\hline $3^{\prime}$ & & $145.5(\mathrm{~s})$ & & & \\
\hline
\end{tabular}


Considering the potent cytotoxic activity of some isoflavonoids, the cytotoxicity of these new isoflavonoids (14) was evaluated against five human cancer cell lines (HL60, A-549, SMMC-7721, MCF-7, and SW-480) using the MTS method. DDP (cisplatin) and paclitaxel were used as positive controls. The results showed that $\mathbf{1}$ and $\mathbf{4}$ exhibited weak cytotoxic activity (Table 5), while $\mathbf{2}$ and $\mathbf{3}$ were inactive $\left(\mathrm{IC}_{50}\right.$ values $\left.>40 \mu \mathrm{M}\right)$ for all cell lines.

\section{Experimental Section}

\subsection{General Experimental Procedures}

Optical rotations were measured on Jasco P-1020 automatic digital polarimeter. $\mathrm{CD}$ spectra were recorded on a Chirascan spectropolarimeter (Applied Photophysics, Leatherhead, Surrey, UK). UV data were obtained from HPLC online analysis. IR spectra were obtained on a Bruker Tensor-27 infrared spectrophotometer with $\mathrm{KBr}$ pellets. NMR spectra were carried out on a Bruker Avance III 600 or DRX-500 spectrometer with deuterated solvent signals used as internal standards. ESIMS and HRESIMS were measured using Agilent G6230 time-of-flight mass spectrometer. Preparative MPLC was performed on a Büchi apparatus equipped with Büchi fraction collector C-660, Büchi pump module C-605 and manager C-615. Silica gel (200-300 mesh, Qingdao Marine Chemical Inc., China), MCI gel CHP-20P (75-150 $\mu \mathrm{m}$, Mitsubishi Chemical Corporation, Japan), Chromatorex C-18 (40-75 $\mu \mathrm{m}$, Fuji Silysia Chemical Ltd., Japan) and Sephadex LH-20 (GE Healthcare Bio-Sciences AB,

Table 5 Cytotoxic activities of compounds 1-4 against five human cancer cell lines

\begin{tabular}{lcllll}
\hline Compd. & HL-60 & A-549 & SMMC-7721 & MCF-7 & SW-480 \\
\hline $\mathbf{1}$ & 19.27 & $>40$ & 19.11 & 39.72 & $>40$ \\
$\mathbf{4}$ & 20.60 & 18.77 & 25.25 & 24.30 & 24.53 \\
DDP & 1.49 & 22.30 & 18.60 & 30.10 & 20.50 \\
Paclitaxel & $<0.008$ & $<0.008$ & $<0.008$ & $<0.008$ & $<0.008$
\end{tabular}

Uppsala, Sweden) were used for column chromatography. Fractions were monitored and analyzed using TLC, in combination with an Agilent 1200 series HPLC system equipped by an Extend-C18 column $(5 \mu \mathrm{m}$, $4.6 \times 150 \mathrm{~mm})$.

\subsection{Plant Material and Isolation (Table 6)}

The retention times $\left(t_{\mathrm{R}}\right)$ of 1-4 on an analytical HPLC Extend-C18 column $\left(20 \% \rightarrow 100 \% \mathrm{MeOH}\right.$ in $\mathrm{H}_{2} \mathrm{O}$ over $8.0 \mathrm{~min}$ followed by $100 \% \mathrm{MeOH}$ to $13.0 \mathrm{~min}, 1.0 \mathrm{~mL} /$ $\min , 25^{\circ} \mathrm{C}$ ) were $7.51,6.85,6.61$ and $9.12 \mathrm{~min}$, respectively.

\subsubsection{7,4'-Dihydroxy-8,2',3'-trimethoxyisoflavan (1)}

White amorphous powder; UV (MeOH) $\lambda_{\max }: 228(\mathrm{sh})$, $278 \mathrm{~nm} ;[\alpha]_{\mathrm{D}}^{25}+11.0(c 0.20, \mathrm{MeOH}) ; \mathrm{IR}(\mathrm{KBr}) v_{\max }: 3396$, 2944, 1602, 1497, 1464, 1292, 1198, 1170, 1097, 1068, 1040, 1010, $963 \mathrm{~cm}^{-1} ;{ }^{1} \mathrm{H}$ NMR data: see Table $1 ;{ }^{13} \mathrm{C}$ NMR data: see Table 2; ESIMS (pos.): $m / z 355[\mathrm{M}+\mathrm{Na}]^{+}$; HRESIMS (pos.): $\mathrm{m} / \mathrm{z} 355.1163[\mathrm{M}+\mathrm{Na}]^{+}$(calcd. for $\mathrm{C}_{18} \mathrm{H}_{20} \mathrm{O}_{6} \mathrm{Na}, 355.1158$ ).

\subsubsection{7,2', 4'-Trihydroxy-8,3'-dimethoxyisoflavan (2)}

White amorphous powder; UV (MeOH) $\lambda_{\max }: 229(\mathrm{sh})$, $277 \mathrm{~nm} ;[\alpha]_{\mathrm{D}}^{25}-13.2(c 0.20, \mathrm{MeOH})$; IR $(\mathrm{KBr}) v_{\max }: 3404$, 2929, 1619, 1509, 1470, 1328, 1184, 1091, 1058, 1037, 1001, $804 \mathrm{~cm}^{-1}$; ${ }^{1} \mathrm{H}$ NMR data: see Table $1 ;{ }^{13} \mathrm{C}$ NMR data: see Table 2; ESIMS (pos.): $m / z \quad 341[\mathrm{M}+\mathrm{Na}]^{+}$; HRESIMS (pos.): $\mathrm{m} / \mathrm{z} 341.1002[\mathrm{M}+\mathrm{Na}]^{+}$(calcd. for $\left.\mathrm{C}_{17} \mathrm{H}_{18} \mathrm{O}_{6} \mathrm{Na}, 341.1001\right)$.

\subsubsection{7,2',4'-Trihydroxy-5-methoxyisoflavan (3)}

White amorphous powder; UV (MeOH) $\lambda_{\text {max }}: 230$ (sh), $279 \mathrm{~nm} ;[\alpha]_{\mathrm{D}}^{25}-24.0($ c $0.20, \mathrm{MeOH})$; IR $(\mathrm{KBr}) v_{\max }: 3406$, 2937, 1620, 1606, 1520, 1501, 1472, 1459, 1200, 1142, 1118, 1037, 974, $819 \mathrm{~cm}^{-1}$; ${ }^{1} \mathrm{H}$ NMR data: see Table 1; ${ }^{13} \mathrm{C}$ NMR data: see Table 2; ESIMS (pos.): $\mathrm{m} / \mathrm{z} 289$ $[\mathrm{M}+\mathrm{H}]^{+}$; HRESIMS (pos.): $m / z 289.1075[\mathrm{M}+\mathrm{H}]^{+}$(calcd. for $\left.\mathrm{C}_{16} \mathrm{H}_{17} \mathrm{O}_{5}, 289.1076\right)$.

Table 6 Plant material and isolation

\begin{tabular}{|c|c|c|c|c|c|c|c|}
\hline Compd. no. & Family & Species & Plant parts & Place of origin & Plant wt. (kg) & Extract wt. (g) & Compd. wt. (mg) \\
\hline 1 & \multirow{2}{*}{ Leguminosae } & \multirow{2}{*}{ S. suberectus } & \multirow{2}{*}{ stems } & \multirow{2}{*}{ Kunming, Yunnan, CN } & \multirow{2}{*}{10.0} & \multirow{2}{*}{800} & 63 \\
\hline 2 & & & & & & & 10 \\
\hline 3 & Leguminosae & F. macrophylla & twigs and leaves & Yuanyang, Yunnan, CN & 10.0 & 930 & 56 \\
\hline 4 & Moraceae & C. cochinchinensis & twigs and leaves & Puer, Yunnan, CN & 13.0 & 1000 & 58 \\
\hline
\end{tabular}




\subsubsection{Cudraisoflavone $M(4)$}

Yellowish amorphous powder; UV (MeOH) $\lambda_{\max }: 212$, 270, 346 (sh) nm; IR (KBr) $v_{\max }: 3425,2926,1644,1582$, 1522, 1435, 1380, 1306, 1209, 1116, 1083, $1030 \mathrm{~cm}^{-1} ;{ }^{1} \mathrm{H}$ and ${ }^{13} \mathrm{C}$ NMR data: see Table 4; ESIMS (pos.): $\mathrm{m} / \mathrm{z} 399$

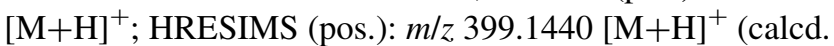
for $\left.\mathrm{C}_{22} \mathrm{H}_{23} \mathrm{O}_{7}, 399.1444\right)$.

\subsection{Cytotoxicity Assays}

Five human tumor cell lines (HL-60, A-549, SMMC-7721, MCF-7, and SW-480) obtained from ATCC (Manassas, VA, USA) were used in the cytotoxicity assay. All cells were cultured in RPMI-1640 or DMEM medium (Hyclone, Logan, UT, USA), supplemented with $10 \%$ fetal bovine serum (Hyclone) at $37^{\circ} \mathrm{C}$ in a humidified atmosphere containing $5 \% \mathrm{CO}_{2}$. Cell viability was assessed by conducting colorimetric measurements of the amount of insoluble formazan formed in living cells based on the reduction of MTS (Sigma, St. Louis, MO, USA). Briefly, $100 \mu \mathrm{L}$ of adherent cells were seeded into each well of a 96-well cell culture plate and allowed to adhere for $12 \mathrm{~h}$ before drug addition, while suspended cells were seeded just before drug addition, both with an initial density of $1 \times 10^{5}$ cells $/ \mathrm{mL}$ in $100 \mu \mathrm{L}$ medium. Each cell line was exposed to the test compound at various concentrations in triplicate for $48 \mathrm{~h}$, with cisplatin and paclitaxel as positive controls. After the incubation, $20 \mu \mathrm{L}$ MTS and $100 \mu \mathrm{L}$ medium was added to each well after removal of $100 \mu \mathrm{L}$ medium, and the incubation continued for $2-4 \mathrm{~h}$ at $37^{\circ} \mathrm{C}$. The optical density was measured at $492 \mathrm{~nm}$ using a Multiskan FC plate reader (Thermo Scientific, USA). The $\mathrm{IC}_{50}$ value of each compound was calculated according to the Reed and Muench method.

\footnotetext{
Acknowledgements This work was financially supported by the "Large-scale Compound Library" project of National Development and Reform Commission of China.
}

\section{Compliance with Ethical Standards}

Conflict of interest The authors declare no conflict of interest.

Open Access This article is distributed under the terms of the Creative Commons Attribution 4.0 International License (http:// creativecommons.org/licenses/by/4.0/), which permits unrestricted use, distribution, and reproduction in any medium, provided you give appropriate credit to the original author(s) and the source, provide a link to the Creative Commons license, and indicate if changes were made.

\section{References}

1. N.C. Veitch, Nat. Prod. Rep. 24, 417-464 (2007)

2. J. Reynaud, D. Guilet, R. Terreux, M. Lussignol, N. Walchshofer, Nat. Prod. Rep. 22, 504-515 (2005)

3. E.E. Shults, M.M. Shakirov, M.A. Pokrovsky, T.N. Petrova, A.G. Pokrovsky, P.G. Gorovoy, Nat. Prod. Res. 31, 445-452 (2017)

4. F. Li, S. Awale, Y. Tezuka, S. Kadota, Bioorg. Med. Chem. 16, 5434-5440 (2008)

5. H.D.T. Mai, T.T.O. Nguyen, V.C. Pham, M. Litaudon, F. Guéritte, D.T. Tran, V.H. Nguyen, Planta Med. 76, 1739-1742 (2010)

6. M.Q. Fu, S.X. Feng, N.L. Zhang, X.J. Zhou, R.M. Huang, H.J. Huang, Z.F. Xu, X.E. Li, S.X. Qiu, Helv. Chim. Acta 95, 598-605 (2012)

7. B. Bueno-Silva, S.M. Alencar, H. Koo, M. Ikegaki, G.V.J. Silva, M.H. Napimoga, P.L. Rosalen, J. Agric. Food Chem. 61, 4546-4550 (2013)

8. N.T. Hiep, J. Kwon, D.W. Kim, B.Y. Hwang, H.J. Lee, W. Mar, D. Lee, Phytochemistry 111, 141-148 (2015)

9. F. Wang, F.C. Ren, Y.J. Li, J.K. Liu, Chem. Pharm. Bull. 58, 1267-1270 (2010)

10. F. Wang, Y.J. Li, F.C. Ren, G.Z. Wei, J.K. Liu, Chem. Pharm. Bull. 59, 484-487 (2011)

11. F. Wang, M.F. Mao, G.Z. Wei, Y. Gao, F.C. Ren, J.K. Liu, Phytochemistry 95, 428-435 (2013)

12. F. Wang, X.L. Li, G.Z. Wei, F.C. Ren, J.K. Liu, Nat. Prod. Bioprospecting 3, 238-242 (2013)

13. G.Z. Wei, M.F. Mao, X.M. Li, F.C. Ren, F. Wang, Nat. Prod. Bioprospecting 6, 111-116 (2016)

14. Y. Goda, F. Kiuchi, M. Shibuya, U. Sankawa, Chem. Pharm. Bull. 40, 2452-2457 (1992)

15. Y. Gao, Y.D. Fang, P. Hai, F. Wang, J.K. Liu, Nat. Prod. Bioprospecting 3, 250-255 (2013)

16. D. Slade, D. Ferreira, J.P.J. Marais, Phytochemistry 66, 2177-2215 (2005)

17. A. Subarnas, Y. Oshima, H. Hikino, Phytochemistry 30, 2777-2780 (1991)

18. W.H. Chen, R. Wang, Y.P. Shi, J. Nat. Prod. 73, 1398-1403 (2010)

19. Y. Hata, S.N. Ebrahimi, M. De Mieri, S. Zimmermann, T. Mokoka, D. Naidoo, G. Fouche, V. Maharaj, M. Kaiser, R. Brun, O. Potterat, M. Hamburger, Fitoterapia 93, 81-87 (2014)

20. W.D. Ollis, I.O. Sutherland, H.M. Alves, O.R. Gottlieb, Phytochemistry 17, 1401-1403 (1978)

21. K. Kurosawa, W.D. Ollis, I.O. Sutherland, O.R. Gottlieb, A.B. De Oliveira, Phytochemistry 17, 1405-1411 (1978)

22. M.A. Ferreira, M. Moir, R.H. Thomson, J. Chem. Soc., Perkin Trans. 1, 2429-2435 (1974)

23. G.F. Spencer, B.E. Jones, R.D. Plattner, D.E. Barnekow, L.S. Brinen, J. Clardy, Phytochemistry 30, 4147-4149 (1991)

24. S. Yahara, T. Ogata, R. Saijo, R. Konishi, J. Yamahara, K. Miyahara, T. Nohara, Chem. Pharm. Bull. 37, 979-987 (1989)

25. H.A.M. Al-Ani, P.M. Dewick, Phytochemistry 24, 55-61 (1985)

26. H.L. Tuan Anh, D.T. Tuan, D.T. Trang, B.H. Tai, N.X. Nhiem, P.H. Yen, P.V. Kiem, C.V. Minh, T.M. Duc, H.K. Kang, Y.C. Kim, Y.H. Kim, J. Asian Nat. Prod. Res. (2016). doi:10.1080/ 10286020.2016.1232253 